\title{
A Model for the Binary Pulsar Radio Eclipse
}

\author{
D. Khechinashvili ${ }^{1,2}$, G. Melikidze ${ }^{1,2}$, and J. Gil ${ }^{1}$ \\ ${ }^{1} J$. Kepler Astronomical Center, Pedagogical University, Lubuska 2, \\ 65-265, Zielona Góra, Poland \\ ${ }^{2}$ Abastumani Astrophysical Observatory, Al.Kazbegi ave. 2a, Tbilisi, \\ 380060 , Georgia
}

\begin{abstract}
We suggest that companions of two binary eclipsing pulsars, B1957+20 and J2051-0827, are degenerate magnetic dwarfs. The magnetospheres of the white dwarfs are infused with relativistic particles supplied by the pulsar wind. We demonstrate that the radio waves emitted by the pulsars are strongly damped due to cyclotron resonance with the plasma particles, in the magnetospheres of companions. Large stable continuos and frequency-dependent radio eclipses should occur as a result of this process. This agrees well with observations.
\end{abstract}

\section{Introduction}

Eclipsing millisecond pulsars were thought to provide the missing link between millisecond pulsars and LMXBs. Observations of at least six low-mass binary systems containing eclipsing MSPs have been reported in the literature. Here we propose a physical mechanism of pulsar radio eclipses in the binary systems PSRs B1957+20 and J2051-0827, on which the richest data is available. The PSR B1957+20 shows regular and entirely periodic eclipses, which occupy about $10 \%$ of its orbital period. Eclipses are quite stable, though their length depends on waves frequency: $55 \mathrm{~min}$ at $318 \mathrm{MHz}$ but $33 \mathrm{~min}$ at $1.4 \mathrm{GHz}$. The eclipse duration of PSR J2051-0827 is also 10\% of it's orbital period and varies between 436 and $660 \mathrm{MHz}$ (Stappers et al. 1996). Eclipse vanishes at higher radio frequencies, so that at $1.4 \mathrm{GHz}$ the pulsar emission is detected throughout the low-frequency eclipse region. The amplitude of the optical counterparts light curve is at least $1.2 \mathrm{mag}$, and the variation in both cases is consistent with the companion's rotating one side being heated by the impinging pulsar flux.

\section{Eclipse mechanism}

Various physical mechanisms of pulsar eclipses were discussed in the literature thus far (see, e.g., Thompson et al. 1994). Fruchter (1995) argued that the companion of PSR $1957+20$ with the mass $m_{c} \sim 0.027 M_{\odot}$ is a degenerate dwarf. We suggest that the same is valid for the companion of PSR J2051-0827 with $m_{c} \sim 0.022 M_{\odot}$. Using known mass-radius relation for white dwarfs one can derive their radii as (depending on the relative concentration of $\mathrm{He}$ and $\mathrm{H}$ 
in their interior) $R_{c} \sim(0.043 \div 0.145) R_{\odot}$. Further, we assume that both white dwarfs possess significant magnetic fields at their surfaces.

The density of pulsar wind plasma at the distances from the pulsar surface corresponding to the binary separations is estimated to be $n_{p} \sim(3.1 \div 3.6) \times$ $10^{-2} \kappa \mathrm{cm}^{-3}$, where $\kappa$ is Sturrock reproduction factor. Here it was assumed that the plasma density falls according to inverse cubic law inside the pulsar light cylinder, and according to the inverse square law beyond it.

We claim that the radio waves emitted by the pulsars split into plasma eigenmodes (ordinary, extraordinary and Alfven waves), as they enter the relativistic plasma of companion's magnetospheres, and are then damped due to cyclotron resonance (Khechinashvili \& Melikidze 1996)

$$
\omega-k_{\varphi} v_{\varphi}-k_{x} u_{d}-\omega_{B} / \gamma_{p}=0 .
$$

Here $\omega_{B}=(e B / m c)$ is Larmor frequency and $\gamma_{p}$ is a mean Lorentz factor of the plasma particles. It is obvious that the angles $\theta$ between wavevectors $\mathbf{k}$ and the local magnetic field $\mathbf{B}$ cannot be small, hence $\theta \gg 1 / \gamma_{p}$. In this approximation we can write the frequency of all damped wavemodes in the form

$$
\nu_{d} \sim 2.8 \times 10^{-3} B_{c}\left(\gamma_{p}(1-\cos \theta)\right)^{-1}\left(R_{c} / r\right)^{3} \quad[\mathrm{GHz}] .
$$

Here $r$ is a distance from the companion's center, $B_{c}$ is the surface magnetic field of the star. Note that we assume this magnetic field to be dipolar. It is seen from Eq.(2) that the frequency of damped waves is inverse proportional to the cube of the distance from the companion's surface (obviously, this dependence results from $\nu_{d} \propto \omega_{B e} \propto B_{e}$, where $B_{e}$ is the magnetic field value in the eclipse region). Therefore, distinct frequencies are damped at distinct heights from the stellar surface (corresponding to appropriate values of the magnetic field), while higher frequencies are damped closer to the companion star. The waves with frequencies somewhat higher and lower than the one given by Eq.(2) propagate almost freely in the plasma at this distance from the star. Although, they can also reach a region at a different altitude or with different direction of local magnetic field (hence, angle $\theta$ ) and be damped there. Thus, the size of "eclipsing spot" is frequency-dependent, being larger at lower frequencies. Indeed, radio waves with a low frequency $\nu_{1}$ (from the entire range of the pulsar radio spectrum) are damped at some large distance $r_{1}$ from the white dwarf surface. At the same time, waves with higher frequency $\nu_{2}>\nu_{1}$ propagate almost freely through this outer region, although they are damped as they reach the distance $r_{2}<r_{1}$ from the stellar surface with a higher value of magnetic field.

Acknowledgments. This paper is supported in part by the KBN grant 2 P03D 015 12. Participation of GM and DK in the Colloquium was supported by the grants from INTAS 96-0154 and Deutsche Forschungsgemeinschaft.

\section{References}

Fruchter, A. S., Stinebring, D. R., \& Taylor, J. H. 1988, Nature, 333, 237

Khechinashvili, D. G., \& Melikidze, G. I. 1997, A\&A, 320, L45

Stappers, B. W., et al. 1996, ApJ, 465, L119

Thompson, C., Blandford, R., Evans, C., \& Phinney, E. 1994, ApJ, 422, 304 